Remark. Admittedly, these two suggestions for evaluating the infinite series might, for some readers, not qualify as proof. For the sceptical reader we recommend the exercise of evaluating the infinite series as a partial fraction expansion. In fact, by analogy with the elementary, real analysis derivation of $\sum_{k=-\infty}^{\infty} 1 /(k \pi+x)^{2}=1 / \sin ^{2} x$ presented in [3, p. 198], we get

$$
\begin{aligned}
\sum_{k=-\infty}^{\infty} \frac{\epsilon}{\epsilon^{2}+(k \pi+x)^{2}} & \leftarrow \frac{1}{n} \sum_{k=-n / 2}^{n / 2-1} \frac{\sinh (2 \epsilon / n) / 2}{\sinh ^{2}(\epsilon / n)+\sin ^{2}((k \pi+x) / n)} \\
& =\frac{\sinh (2 \epsilon) / 2}{\sinh ^{2} \epsilon+\sin ^{2} x}
\end{aligned}
$$

as $n=2^{v} \rightarrow \infty$. Here, the identity follows recursively from the simple case $v=1$. A little endurance in massaging the hyperbolic and trigonometric functions helps us to conclude the proof with the identity

$$
\frac{1}{1+\eta^{2}} \cdot \frac{\sinh (2 \epsilon) / 2}{\sinh ^{2} \epsilon+\sin ^{2}(\arctan \eta)}=\frac{\tanh \epsilon}{\tanh ^{2} \epsilon+\eta^{2}} .
$$

ACKNOWLEDGMENTS This note was inspired by Brian Davies, who challenged Nick Trefethen at Oxford by pointing out that the evaluation of the integral (7) would cause serious difficulties both analytically and numerically. One of the authors (T.S.) had the pleasure of attending Trefethen's course in Oxford, where problems like Davies's had to be solved on a weekly basis. Discussing the integral with Christoph Ortner was helpful, too. T.S. is supported by a Rhodes Scholarship.

\title{
REFERENCES
}

1. F. Bornemann, D. Laurie, S. Wagon, and J. Waldvogel, The SIAM 100-Digit Challenge: A Study in HighAccuracy Numerical Computing, SIAM, Philadelphia, 2004.

2. P. Henrici, Applied and Computational Complex Analysis, vol. 3, Wiley, New York, 1986.

3. J. Hofbauer, A simple proof of $1+\frac{1}{2^{2}}+\frac{1}{3^{2}}+\cdots=\frac{\pi^{2}}{6}$, this MONTHLY 109 (2002) 196-200.

4. A. Prudnikov, Y. Brychkov, and O. Marichev, Integrals and Series. Vol. 1: Elementary Functions, Gordon \& Breach, New York, 1986.

5. W. Rudin, Real and complex analysis, 3rd ed., McGraw-Hill, New York, 1987.

Mathematik, Technische Universität München, 80290 München, Germany http://www.ma.tum.de/m3/bornemann/

bornemann@ma.tum.de

Oxford University Computing Laboratory, Wolfson Building, Parks Road, Oxford OX1 3QD, U.K. http://www.comlab.ox.ac.uk thomas.schmelzer@balliol.ox.ac.uk

\section{Pick's Theorem via Minkowski's Theorem}

\section{Ram Murty and Nithum Thain}

In 1899, Georg Alexander Pick published one of his most beautiful theorems [12]. This theorem provided a formula for easily calculating the area of a planar polygon $P$ whose vertices have integer coordinates. Such a polygon is called a lattice polygon, since the 
points in the plane with integer coordinates are sometimes called lattice points. In fact, Pick's theorem states that if $I$ is the number of lattice points in the interior of $P$ and $B$ is the number of lattice points on its boundary, then the area $A$ of $P$ is given by

$$
A=I+\frac{B}{2}-1 \text {. }
$$

The beauty of this formula stems from its simplicity and depth. It has been successfully explained to twelve-year-olds, and yet mathematicians are still researching some of its consequences today.

Pick was born into a Jewish family in Vienna on August 10, 1859. He received his Ph.D. from the University of Vienna under the supervision of Leo Koenigsberger in 1880. He spent most of his working life at the University of Prague, where his colleagues and students praised his excellence at both research and teaching. In 1910, Albert Einstein applied to become a professor of theoretical physics at the University of Prague. Pick found himself on the appointments committee and was the driving force in getting Einstein accepted. For the brief period that Einstein was at Prague, he and Pick were the closest friends. They were both talented violinists and frequently played together. In 1929, Pick retired and moved back to his hometown of Vienna. Nine years later, Austria was annexed by Germany. In an attempt to escape the Nazi regime, Pick returned to Prague. However, on July 13, 1942, he was captured and transported to the Theresienstadt concentration camp. He passed away there thirteen days later, at the age of 82 [8].

Pick's formula first came to popular attention in 1969 (seventy years after Pick published it) in Steinhaus's book Mathematical Snapshots [15]. Since then, mathematicians have come up with a variety of different proofs using tools ranging from Euler's formula to the Weierstrass $\wp$-function [1], [2], [4], [9], [16]. We have also seen important connections between this formula (and its generalization) and ideas in combinatorics, algebraic geometry, and complex analysis [2], [4].

In this note, we present a new proof of Pick's theorem via Minkowski's convex body theorem. We begin by reviewing Minkowski's theorem for the benefit of the reader (see [10] or [11, p. 76] giving Siegel's proof of it). Recall that a region $R$ is an open, connected set; $R$ is a symmetric region if $x$ in $R$ implies that $-x$ is in $R$.

Theorem (Minkowski's Convex Body Theorem). A bounded, symmetric, convex region $C$ in $\mathbb{R}^{n}$ with volume greater than $2^{n}$ contains at least one nonzero lattice point.

Proof. We first define a function $\varphi$ on $\mathbb{R}^{n}$ by $\varphi(x)=1$ if $x$ belongs to $C / 2=\{y / 2$ : $y \in C\}$ and $\varphi(x)=0$ otherwise. We then set

$$
\Phi(x)=\sum_{\lambda \in \mathbb{Z}^{n}} \varphi(x+\lambda) .
$$

The function $\Phi$ is bounded and integrable, so

$$
\begin{aligned}
\int_{[0,1]^{n}} \Phi(x) d x & =\int_{[0,1]^{n}} \sum_{\lambda \in \mathbb{Z}^{n}} \varphi(x+\lambda) d x=\int_{\mathbb{R}^{n}} \varphi(x) d x \\
& =\operatorname{vol}\left(\frac{C}{2}\right)=\frac{\operatorname{vol}(C)}{2^{n}}>1 .
\end{aligned}
$$

Since $\Phi$ is integer-valued, we must have $\Phi(x) \geq 2$ for some $x$. In other words, there are two distinct points $P+\lambda_{1}$ and $P+\lambda_{2}$ in $C / 2$ that differ by a lattice point. Call 
these points $P_{1} / 2$ and $P_{2} / 2$ (so $\left(P_{1}-P_{2}\right) / 2$ is a lattice point). Then $P_{1}$ and $P_{2}$ are in $C$, and by symmetry and convexity, $\left(P_{1}-P_{2}\right) / 2$ is also in $C$. Thus $\left(P_{1}-P_{2}\right) / 2$ is a nonzero lattice point in $C$.

To avoid confusion later, it is appropriate to fix some definitions at this point. By a polygon in the plane, we mean a compact subset of $\mathbb{R}^{2}$ whose boundary consists of a finite number of straight line segments that form a single non-self-intersecting cycle. A convex polygon is a polygon such that for any two points in the polygon the line segment connecting them also lies entirely in the polygon. If $P_{1}$ and $P_{2}$ are two polygons in the plane that share portions of their boundaries but are otherwise disjoint and if $P=P_{1} \cup P_{2}$, then we say that $P$ can be decomposed into $P_{1}$ and $P_{2}$. We have indicated earlier what a lattice polygon is.

In trying to prove Pick's theorem, we will need to "triangulate" a convex lattice polygon. To do so, we take any vertex and connect it to all other vertices by straight line segments (some of which may already be edges of the polygon). In this way, we decompose the polygon into triangles. Furthermore, any lattice point in the interior of such a triangle $T$ can be joined to the vertices of the triangle, while any lattice point on an edge of $T$ can be joined to the opposite vertex. In this way we can decompose a lattice polygon into the union of lattice triangles that contain no lattice points apart from vertices. We call such a triangle an elementary triangle.

We now use Minkowski's theorem to prove Pick's theorem for the case of elementary triangles. The key to this proof is constructing a bounded, symmetric, convex figure with no nonzero lattice points out of eight copies of a given elementary triangle.

Lemma. Every elementary triangle has area $1 / 2$.

Proof. Let $\triangle A B C$ be an elementary triangle. Rotate this triangle by 180 degrees around the vertex $A$, and call the new triangle obtained in this way $\Delta A_{1} B_{1} C_{1}$ (where $B_{1}$ is the image of $B$, and so on). We can translate this new triangle by a lattice point so that side $B_{1} C_{1}$ attaches to side $C B$ of the old triangle. Note that both the rotation and translation are invertible transformations that send lattice points to lattice points. Since the only lattice points in $\triangle A B C$ were the vertices, there will be no lattice points in the interior of this new figure.

If we repeat the foregoing procedure for vertices $B$ and $C$ we end up with a triangle (call it $P$ ). We notice that the there are no lattice points in the interior of $P$. Now, if we rotate $P$ by 180 degrees about $B$ and attach this to our original $P$, then the resulting figure is a bounded, symmetric, convex figure (Figure 1).

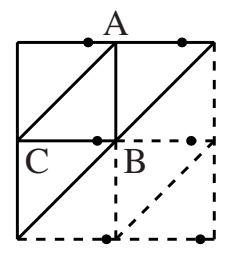

Figure 1.

The only lattice point in the interior of this figure is $B$, which we may take to be the origin. By Minkowski's theorem, this figure has area no larger than 4 . The area of this figure is eight times the area of $\triangle A B C$, so $\triangle A B C$ has area at most $1 / 2$. 
Now let $\left(x_{1}, y_{1}\right),\left(x_{2}, y_{2}\right)$, and $\left(x_{3}, y_{3}\right)$ be the coordinates of $A, B$, and $C$, respectively. Then the area of $\triangle A B C$ is the absolute value of

$$
\frac{1}{2}\left|\begin{array}{lll}
x_{1} & y_{1} & 1 \\
x_{2} & y_{2} & 1 \\
x_{3} & y_{3} & 1
\end{array}\right| .
$$

Because the determinant here is a nonzero integer, this expression is always at least $1 / 2$.

We can now prove Pick's theorem in general, by showing that the formula $I+B / 2-1$ is additive and decomposing a general polygon into elementary triangles.

Theorem (Pick's Theorem). Suppose that $P$ is a convex lattice polygon. If $B$ is the number of lattice points on the boundary of $P$ (including vertices) and $I$ is the number of lattice points in the interior of $P$, then the area of $P$ is given by the expression $I+B / 2-1$.

Proof. The lemma establishes that every elementary triangle satisfies this formula. Now suppose that the lattice polygon can be decomposed into two lattice polgyons $P_{1}$ and $P_{2}$ for which the Pick formula holds. We show that $P$ must satisfy this formula as well. Let $I_{X}$ and $B_{X}$ signify the number of lattice points in the interior and boundary, respectively, of a planar compact set $X$. Note that

$$
I_{P}=I_{P_{1}}+I_{P_{2}}+D-2,
$$

where $D$ is the number of points on the common boundary of $P_{1}$ and $P_{2}$. Also,

$$
B_{P}=B_{P_{1}}+B_{P_{2}}-2 D+2 \text {. }
$$

From this it follows that

$$
\begin{aligned}
I_{P}+\frac{B_{P}}{2}-1 & =I_{P_{1}}+\frac{B_{P_{1}}}{2}-1+I_{P_{2}}+\frac{B_{P_{2}}}{2}-1 \\
& =\text { area of } P_{1}+\text { area of } P_{2}=\text { area of } P .
\end{aligned}
$$

Since any lattice polygon can be decomposed into elementary triangles, the proof is complete.

Minkowski's theorem is true for spaces of any dimension, and this leads us to hope that we might be able to generalize Pick's theorem to higher dimensions. However, there is no simple generalization of Pick's theorem even to three dimensions. To see this, notice that the tetrahedron with vertices $(0,0,0),(1,0,0),(1,1,0)$, and $(0,1, r)$ is an elementary tetrahedron for all positive integers $r$ but has volume $r / 6$. Thus, we cannot write down a formula for the volume of a polyhedron in terms of integer lattice points.

J. E. Reeve was able to generalize Pick's theorem to three dimensions with the help of auxiliary lattices [13]. One such auxilliary lattice is the lattice of points $(a, b, c)$ in $\mathbb{R}^{3}$ such that $(2 a, 2 b, 2 c)$ is in $\mathbb{Z}^{3}$. Reeve was able to calculate the volume of a lattice polyhedron by counting the number of integer lattice points and auxilliary lattice points in the interior and boundary of that polyhedron. 
Generalizations to even higher dimensions are possible by way of so-called Ehrhart polynomials [6], [7], [14]. If $P$ is a $d$-dimensional polytope in $\mathbb{R}^{d}$, then define $F_{P}(n)=$ \#\{lattice points in $n P\}$. It is a theorem of Ehrhart that $F_{P}(n)=a_{d} n^{d}+a_{d-1} n^{d-1}+$ $\cdots+a_{0}$ is a polynomial in $n$ of degree $d$, called the Ehrhart polynomial of $P$. Ehrhart was also able to determine some of the coefficients of this polynomial. He proved that $a_{d}=\operatorname{vol}(P)$ is the volume of $P, a_{d-1}=(1 / 2) \operatorname{vol}(\delta P)$ is half the surface area of $P$ normalized with respect to the sublattice on each facet of $P$, and $a_{0}$ is the Euler characteristic of $P$. Note that in the case of a two-dimensional polytope in $\mathbb{R}^{2}$, we can retrieve Pick's formula by evaluating $F_{P}(1)=\operatorname{vol}(P)+B / 2+1$ and noting that $F_{P}(1)=I+B$ in the notation of the paper. The other coefficients of the polynomial remained mysterious for some time. Recently, Morelli and others have been able to link the other coefficients to Todd classes of toric varieties [3], [5].

ACKNOWLEDGMENTS We thank the referee and Mike Roth for their comments and suggestions that improved the readability of the paper. The first author's research is partially supported by an NSERC grant. The seond author's research is supported by an NSERC Undergraduate Student Award.

\section{REFERENCES}

1. C. Blatter, Another proof of Pick's theorem, Math. Mag. 70 (1997) 200.

2. A. Bogomolny, A proof of Pick's theorem, "Cut-the-Knot" website, http://www . cut-the-knot.org/ ctk/Pick_proof.shtml.

3. M. Brion and M. Vergne, Lattice points in simple polytopes, J. Amer. Math. Soc. 10 (1997) 371-392.

4. R. Diaz and S. Robins, Pick's formula via the Weierstrass $\wp$-function, this MonTHLY 102 (1995) 431437.

5. - The Ehrhart polynomial of a lattice polytope, Annals of Math. 145 (1997) 503-518.

6. E. Ehrhart, Sur un problème de géométrie diophantienne linéaire I, J. Reine Angew. Math. 226 (1967) 25-49.

7. —— Sur un problème de géométrie diophantienne linéaire II, J. Reine Angew. Math. 227 (1967) 25-49.

8. R. Fritsch, Georg Pick und Ludwig Berwald—zwei Mathematiker an der Deutschen Universität in Prag, Schr. Sudet.dtsch Akad. Wiss. Künste Forsch.beitr. Nat.wiss. 22 (2001) 9-16.

9. W. W. Funkenbusch, From Euler's formula to Pick's formula using an edge theorem, this MonTHLY 81 (1974) 647-648.

10. H. Minkowski, Geometrie der Zahlen, Teubner, Leipzig-Berlin (1896); reprinted by Chelsea, New York, 1953.

11. M. R. Murty and J. Esmonde, Problems in Algebraic Number Theory, 2nd ed., Springer-Verlag, New York, 2005.

12. G. Pick, Geometrisches zur Zahlentheorie, Sitzungber. Lotos (Prague) 19 (1899) 311-319.

13. J. E. Reeve, On the volume of lattice polyhedra, Proc. London Math. Soc. 7 (1957) 378-395.

14. R. Stanley, Enumerative Combinatorics, vol. 1, Cambridge University Press, Cambridge, 1996.

15. H. Steinhaus, Mathematical Snapshots, Dover, New York, 1999.

16. D. E. Varberg, Pick's theorem revisited, this MonThLY 92 (1985) 584-587.

Department of Mathematics, Jeffery Hall, 99 University Avenue, Queen's University, Kingston, Ontario, K7L 3N6, Canada 